\title{
Computational methods and applications for quantitative systems pharmacology
}

\author{
Fuda $\mathrm{Xie}^{1,2}$, Jiangyong $\mathrm{Gu}^{1,2, *}$ \\ 1 The Second Clinical College, Guangzhou University of Chinese Medicine, Guangzhou 510006, China \\ 2 Guangdong Provincial Academy of Chinese Medical Sciences, Guangzhou 510006, China \\ * Correspondence: gujy@gzucm.edu.cn
}

Received June 29, 2018; Revised August 8, 2018; Accepted August 31, 2018

\begin{abstract}
Background: Quantitative systems pharmacology (QSP) is an emerging discipline that integrates diverse data to quantitatively explore the interactions between drugs and multi-scale systems including small compounds, nucleic acids, proteins, pathways, cells, organs and disease processes.

Results: Various computational methods such as ADME/T evaluation, molecular modeling, logical modeling, network modeling, pathway analysis, multi-scale systems pharmacology platforms and virtual patient for QSP have been developed. We reviewed the major progresses and broad applications in medical guidance, drug discovery and exploration of pharmacodynamic material basis and mechanism of traditional Chinese medicine.

Conclusion: QSP has significant achievements in recent years and is a promising approach for quantitative evaluation of drug efficacy and systematic exploration of mechanisms of action of drugs.
\end{abstract}

Keywords: quantitative systems pharmacology; network modeling; multi-scale platforms; traditional Chinese medicine

\begin{abstract}
Author summary: Quantitative systems pharmacology (QSP) is an emerging discipline that integrates diverse data to quantitatively explore the interactions between drugs and multi-scale systems including small compounds, nucleic acids, proteins, pathways, cells, organs and disease processes. This review is an attempt to introduce the computational methods for QSP, including ADME/T (absorption, distribution, metabolism, excretion and toxicity) evaluation, molecular modeling, logical modeling, network modeling, pathway analysis, multi-scale systems pharmacology platforms and virtual patient as well as their applications in medical guidance, drug discovery and explorations of pharmacodynamics material basis and mechanism of traditional Chinese medicine.
\end{abstract}

\section{INTRODUCTION}

Systems pharmacology (SP) combines systems biology approaches and computational methods to enable drug discovery for complex diseases and understand mechanisms of action (MoA) of drugs [1-4]. SP provides holistic approaches to facilitate the prediction of effectiveness and safety of molecules during the process of drug discovery. The human body is a complicated and integrated system, which can be regarded as biological networks [5]. Methods that can be applied to quantitative evaluation of the complex interactions between drugs and diseaserelated systems are urgently needed. The etiology and pathogenesis of complex diseases such as cancer, schizophrenia, and Alzheimer's disease concern lots of genes, gene products, small molecules and pathways, and there are still challenges in disease treatment [6-10]. Quantitative systems pharmacology (QSP), as a branch of $\mathrm{SP}$, is an emerging approach to understand the interaction mechanism between drugs and the body and to predict the pharmacological effects of drugs [11-13]. QSP integrates diverse data, including preclinical and clinical information to analyze dynamic interactions between a drug or drug combination and multi-scale biological systems, that aims to understand the behavior of the systems as a whole $[14,15]$. It can also provide quantitative insights into biological and pharmacological processes [16]. QSP gets more and more attention in pharmacological research and 
pharmaceutical industry $[13,17,18]$.

QSP is usually described as three steps: gathering enough information such as disease-related targets, biomarkers, pathways, drug-target interactions and phenotypic characteristics; building a primary model based on the above information, calibrating and validating the model by comparing predictions with preclinical and clinical data $[19,20]$. QSP is a promising approach to quantitatively explore the interactions between drugs and the systems including targets, pathways, cells and organs and provides a comprehensive insight into the underlying mechanisms of drug action [21].

QSP has been becoming a discipline and the research in QSP involves describing pharmacokinetic/pharmacodynamic (PK/PD) characteristics of drugs, identifying the targets and drug-target interactions and investigating the factors that cause differences in the omics data of cells, tissues and patients [22,23]. Traditional drug discovery holds the thought "one drug, one target, one disease" and tries to treat the disease by adjustment of a single target which is responsible for the disease. This simple strategy pushes drug design to focus on selective drugs for specific targets. With the in-depth understanding of biological processes and pathogenesis, the disease phenotypes often represent a complex regulating network with multiple targets, pathways and cell signal transduction [1,24-29]. A single target can also be related directly or indirectly with many kinds of diseases [30,31]. QSP integrates the understanding of complex networks of diseases and adopts quantitative analytical and predictive methods, which provides a feasible approach for the development of new multi-target drugs and exploration of their MoAs. This review is an attempt to introduce the computational methods and applications for QSP, including ADME/T (absorption, distribution, metabolism, excretion and toxicity) prediction, network pharmacology and multiscale systems pharmacology platforms (Table 1).

\section{METHODS FOR QSP}

\section{Molecular-level evaluation and simulation}

At the molecular level, QSP focuses on the evaluation of molecular properties and identification of drug-target interactions. These methods such as ADME/T analysis, chemical space analysis, drug-likeness evaluation can provide information about the characteristics of metabolism of drugs or compounds. The widely used PK/PD models provide the most basic data about drug absorption, distribution, metabolism, excretion and toxic characteristics [32,69-71]. PK and PD data indicate how drugs change in vivo over time and the characteristics of targets to elucidate the mechanism of drug action $[14,32-34,69]$. For example, Rostami-Hodjegan took into account the knowledge of physiology and biology based PK to predict the effects of intrinsic and extrinsic factors of drugs [69].

There are several computational methods to simulate the drug-target interaction such as molecular docking, molecualr dynamics simulation, machine learning and similarity analysis. Molecular docking and molecular dynamics simulation are feasible approaches for drug discovery, which give insights into the conformation of drug-target interaction and provide theoretical basis for virtual screening of lead compounds [37,38,72,73]. Rational drug design can be carried out by simulating the characteristics of targets and interactions with drugs

Table 1 Computational methods for QSP

\begin{tabular}{|c|c|c|c|}
\hline & Method classification & Description & Refs. \\
\hline \multirow[t]{2}{*}{ Molecular level } & $\begin{array}{l}\text { Evaluation of molecular } \\
\text { characteristics }\end{array}$ & $\begin{array}{l}\text { Providing information about molecular properties of drugs (ADME/T, PK/PD } \\
\text { model, chemical space analysis and drug-likeness evaluation) }\end{array}$ & {$[1-4,32-36]$} \\
\hline & $\begin{array}{l}\text { Identification of } \\
\text { drug-target interaction }\end{array}$ & $\begin{array}{l}\text { Predicting and evaluating drug/compound-target interaction (molecular docking, } \\
\text { molecular dynamics simulation, machine learning and similarity analysis) }\end{array}$ & {$[37-42]$} \\
\hline \multirow[t]{3}{*}{ Network level } & $\begin{array}{l}\text { Drug-target network } \\
\text { analysis }\end{array}$ & Analyzing the interactions between drugs and targets & {$[43-45]$} \\
\hline & $\begin{array}{l}\text { Protein-protein interaction } \\
\text { network analysis }\end{array}$ & $\begin{array}{l}\text { Analyzing topological structures of complicated protein-protein interaction } \\
\text { network }\end{array}$ & {$[46]$} \\
\hline & Pathway analysis & $\begin{array}{l}\text { Investigating the connections between drug targets and regulatory networks of } \\
\text { diseases, and evaluating drug efficacy in thecontext of pathway network }\end{array}$ & {$[47-50]$} \\
\hline \multirow[t]{3}{*}{ Systems level } & Logical modeling & $\begin{array}{l}\text { A mechanism-based mathematical method to endow the object with logical } \\
\text { structure. }\end{array}$ & {$[51]$} \\
\hline & $\begin{array}{l}\text { Multiscale systems } \\
\text { pharmacology platform }\end{array}$ & \multicolumn{2}{|l|}{$\begin{array}{l}\text { Evaluating the treatment effects of therapeutic regimens and exploring the MoA [52-64] } \\
\text { by integrating preclinical/clinical data of drugs and disease phenotypes (TCMSP, } \\
\text { Virtual Tumour, CancerHSP, etc.) }\end{array}$} \\
\hline & Virtual patient & $\begin{array}{l}\text { A simplified model to translate complex biological processes into a series of } \\
\text { intuitive equations }\end{array}$ & {$[65-68]$} \\
\hline
\end{tabular}


and the high-throughput virtual screening is accomplished by analyzing the binding affinity between compound and target. For example, Omer discovered two novel antiviral molecules (Calanolide A and Chaetochromin B) and their target HRAS by molecular docking and molecular dynamics simulation [73]. In our recent work, a binding energy-weighted polypharmacological index was introduced to evaluate the importance of target-related pathways which had close correlation with the pathogenesis of psoriasis [74].

Machine learning and similarity analysis are another two important approaches to explore drug-target interactions and drug-drug interactions. Machine learning is a method used to improve the performance on a specific model with data, and plays an important role in systems pharmacology [39-42]. For example, Chiu and Xie integrated coarse-grained normal mode analysis with multi-target machine learning to predict protein-ligand binding/unbinding kinetics accurately [41]. Yang et al. constructed three best-performing model to screen inhibitors for P-glycoprotein (P-gp) by machine learning algorithm, and these models were employed as a virtual screening tool for identifying 875 potential P-gp inhibitors and 15 inhibitor-rich herbs from TCMSP [75]. Compounds that have similar structures would have similar functions. Predicting targets for a new molecule by comparing the similarity with active compounds whose targets are known is a traditional method. BindingDB [76] and BATMAN-TCM [77] are two famous web-server which can predict drug-target interaction by analyzing molecular similarity.

\section{Network modeling and pathway analysis}

Network modeling integrates disease-related genes, pathways, targets and drugs into a complex network model and provides frameworks for understanding of how regulation arises from the interactions between cellular components $[1,2,43-45]$. The biological systems can be regarded as networks, where nodes represent molecular entities (DNA, RNA, protein and small compound) and processes, edges represent the relationships between nodes. Important nodes and edges in the network can be identified by network analysis. The change of global characteristics of network can be determined by network dynamics simulation. The network model can provide important information such as key targets in regulatory networks, the mechanism of interactions between drugs and targets. The results of network modeling can provide theoretical basis and guide for the development of multitarget drug, drug combination and credible options for personalized treatment as well as a feasible way to explore the pathogenesis of diseases. These network-based approaches are useful in understanding the basis for cancer combination therapy [3], discovering treatment regimens for optimal efficacy [78], identifying the origins of drug induced adverse events [79-81], and indicating how drug combinations can mitigate serious adverse events [82]. For example, Wu developed an integrated network and cheminformatics tool (SDTNBI) for systematic prediction of drug-target interactions and drug repositioning $[83,84]$. Wang applied network topologies and dynamics parameters to obtain two potential weak-binding drug candidates whose effects were validated by in vitro experiments so as to provide a feasible way for drug discovery [85].

Pathway analysis is an approach to investigate the therapeutic mechanism by analyzing the connections between drug targets and regulatory networks of diseases [47]. It is a universal way that provides various information as the basis of many models in QSP [86]. Topological analysis is usually used to measure the importance of genes to simplify the complex pathway network into a structured collection of related genes. It can effectively reduce the difficulty of modeling and analyzing the pathway network which is responsible for the disease phenotype. But it may reduce the accuracy of the results as it ignores some potentially valuable information such as the connections of genes that belong to different pathways and potential pathogenic genes [87]. Nie et al. studied the regulation mechanism of Toutongning capsule by analyzing the signaling pathway of the migraine and the results showed that 19 active compounds and 8 targets played a crucial role in the treatment of migraine through TNF pathway [88].

We have developed a pathway network-based method by combining network modeling and molecular docking to evaluate drug efficacy. Network efficiency $(N E)$ and network flux $(N F)$ are both global measures of the network connectivity. We used $N E$ and $N F$ to quantitatively evaluate the inhibitory effects of compounds. The edge values of the pathway network were reset according to the Michaelis-Menten equation, which used the binding constant and drug concentration to determine the degree of inhibition of the target protein in the pathway. The dose-response curve was sigmoid and the predicted effects of compounds were in good agreement with experimental results [5,48-50]. Moreover, This approach can be used for predictions of drug combination and drug repositioning $[5,89]$.

\section{Systems-level methods}

\section{Logical modeling}

Logical modeling is a kind of mathematical method based on the mechanism which can endow the object with logical structure. It can provide insights into a variety of 
phenomics profiles through the analysis of the logical relationship between phenotype and mechanism [51,90-92]. This modeling method can be established according to the known information of the biological process and optimized by calibrating the modeling results with experimental data. Then altering parameters of the model to simulate the changes of biosystems to obtain various outcomes which can provide useful information and meaningful predictions to the process. This approach can provide reasonable way to build enormous biological network models in lack of various preclinical and clinical data by predicting the logical relationship. However, it is important to be aware of the simplification in this mechanism-based simulation which would cause the impossibility of representing the complexity and diversity of biological systems [51]. Poltz et al. built a discrete logical model of signal transduction of DNA damage response to screen target proteins for DNA-damaging agents that could be suitable for radio- and chemotherapy, and contributed to the design of more effective therapies [90].

\section{Multi-scale systems pharmacology platforms}

QSP takes the whole body as the starting point of research to seek the relationship between drug administration and disease to speculate the underlying mechanisms. It is also used to guide personalized medicine by integrating genomics knowledges. Multi-scale systems pharmacology focuses on disease-related multiple drugs, targets, pathways, biomarkers and phenomics. Several multi-scale systems pharmacology platforms have been developed such as TCMSP [52], Virtual Tumour [53], CancerHSP [54], C ${ }^{2}$ Maps [55], VisANT 4.0 [56], PDTCM [57], CVDHD [58], Lipoprotein Metabolism and Kinetics (LMK) Platform [59], Rheumatoid Arthritis PhysioLab platform [60], and others [61-64].

TCMSP is a unique systems pharmacology platform of Chinese herbal medicines and sparks a new interest in the search of candidate drugs from TCM [52]. TCMSP contains chemicals and their pharmacokinetic properties, targets and drug-target networks, drug-target-disease networks to capture the relationships between drugs, targets and diseases. Virtual Tumour Preclinical platform integrates available PK and cell cycle PD measurements for chemotherapeutic and targeted cancer treatment agents into a model of cell cycle and xenograft tumor growth [53]. Musante et al. reported an ImmunoOncology (I-O) platform to investigate the effects of two kinds of regimens for cancer and suggest possible applications based on clinical data and analysis of mechanism [59]. Kirouac et al. developed a multi-scale systems model of ErbB signaling to support the preclinical investigation of a bispecific antibody targeting HER2 and HER3 in cancer [93]. Another important QSP modeling platform is the DILIsym ${ }^{\circledR}$ which is developed by the non-profit Hamner Institutes of Health Sciences. It can be used for drug development [94], explaining the mechanisms of hepatotoxicity [95] and liver toxicities [96]. PDTCM [57] and CVDHD [58] are two online servers that developed for psoriasis and cardiovascular disease, respectively. PDTCM and CVDHD integrated medicinal herbs, natural products, disease-related proteins, docking results and clinical biomarkers. By using virtual screening and network pharmacological methods, PDTCM and CVDHD streamline drug/lead discovery from natural products and explore the action mechanism of medicinal herbs and formulae [57,58].

These platforms that combine the preclinical/clinical data of many aspects and a variety of disease phenotypes are able to evaluate the treatment effects of therapeutic regimens and explore the MoA. These models are also applicable to different diseases after appropriate adjustment. However, the development of these platforms requires a certain depth of clinical research on the diseases and a wider range of preclinical and clinical data [59].

\section{Virtual patient}

Using QSP model to translate complex biological processes into a series of intuitive equations is a promising way to get insights into curative effects in drug discovery and disease treatment. However, the preclinical and clinical data for the establishment of models is lacking in some aspects. Many scientists simplified the models by alternative parameterization to reduce the need for data, and this method is also called "virtual patient" [65-68,97]. Moreover, a mechanistically-based weighting method to match clinical trial statistics at population level was introduced in a comprehensive analysis (virtual population) [60]. Geerts et al. used a mechanism-based QSP platform, virtual human patient, to simulate the biological processes of Alzheimer's disease and to build a tool to realize personalized drug treatment [66]. Allen et al. [68] developed a new approach to generate virtual population without the step of weighting. This approach includes following steps: define plausible ranges for model parameters and initialize parameters; calibrate the model by comparing the prediction with database, then repeat the selection and optimization steps until the available model patients are plentiful enough. Finally, a credible virtual population model is constructed after calculating probability of inclusion into virtual population and optimizing the inclusion rate. 


\section{APPLICATIONS}

\section{Drug discovery}

The strategy of drug discovery has been shifting from searching selective drug for single target that aimed to decrease side effects into looking for drugs that can rebalance the biological processes and regulatory networks $[1,25,98-104]$. The in vivo dynamics and kinetics of drug-target interactions can be simulated and evaluated by establishing QSP models to reduce the cost of money and time in a certain extent comparing with the traditional in vivo experiments [78,105]. QSP models integrate multiple regulatory networks of disease-related biological processes and build platforms for screening. The applications of these platforms can increase the efficiency of high-throughput screening of candidate compounds and reduce the time required to study the links between drugs and complex networks. Unwanted side effects and toxicity of candidate drugs can be evaluated by adverse drug reactions [82,106-109] and toxicity models [70,110]. Liu et al. [111] applied a comprehensive systems approach to identify 73 bioactive components from licorice and 91 potential targets for this herb. The mechanism of this herbal medicine by mapping drugtarget and drug-target-disease networks was further elucidated. Luo used a network-based multi-target computational approach to screen potential anticancer drugs from natural products and predict the interactions between anticancer drugs and cancer-related targets [112]. Archimedes model is a human physiology-based statistical disease progression model to simulate the effect of treatments for cardiometabolic diseases $[113,114]$. These works all make it easier and cheaper to find new effective drugs.

\section{Medical guidance}

QSP models are utilized to inform different questions in pharmacology, such as MoA exploration, efficacy evaluation, translational medicine and drug discovery. The QSP modeling of drug metabolizing process usually uses a time dependent equation. QSP models can be built on a time scale while the time of reaction can be as short as action process of quick-acting drugs and as long as the generation and deterioration of chronic diseases. QSP modeling approaches can address challenges in the translation of preclinical findings to the clinical applications [115-117]. Instead of analyzing the instantaneous outputs of models, researchers usually use frequencydomain response analysis in mathematics to explore the process of change under perturbation (treatment of disease) at the systems level [118]. Taylor et al. analyzed 14 distinct PD models of four class (indirect response, auto regulation, precursor-pool and moderator-mediated feedback) to evaluate the practicability of frequencydomain response analysis method [118].

There are also many other kinds of medical guidance provided by QSP models. Visser et al. simulated and optimized in vivo dosing regimens by informing both preclinical and translational evaluation of single drug and combination therapy [119]. Geerts et al. contributed a lot in development of schizophrenia treatment such as predicting the effect of existing drugs and developed a mechanism-based QSP model of a relevant key cortical brain network with schizophrenia pathology to gain insights of cognitive deficits in schizophrenia [97,120 122]. Vega-Villa et al. developed a QSP model to characterize metabolome of nitric oxide after a longterm infusion of sodium nitrite that would be valuable for nitrite dosing selection in clinic [123]. John et al. investigated the mechanisms of anxiolytic drugs on hippocampal electric patterns and interpreted the stimulus-frequency relationship of hippocampal theta [124]. Rostami-Hodjegan developed a physiologically based pharmacokinetic model to guide administration of oseltamivir in pediatric patients $[69,125]$. Recently, Kaddi et al. presented a multiscale and mechanistic QSP modeling of acid sphingomyelinase deficiency and the enzyme replacement therapy that quantitatively assessed systemic pharmacological effects in adult and pediatric patients at molecular-level, cellular-level, and organ-level effects [126]. Other works contributed to expand knowledge of disease processes by phenotypic screening and developing personalized medicine [47,127-129].

\section{QSP in TCM}

Systems pharmacology methods are frequently used in exploration of pharmacodynamic material basis and MoA of traditional Chinese medicine (TCM) [130-139]. The recent applications of these QSP methods in TCM are summarized in Table 2. For example, Li et al. dissected the mechanism of the addition and subtraction theory of traditional Chinese medicine by building a SP platform to contrast and analyze the variation of kinetic parameters and targets of active compounds in Xiao-Chaihu-Decoction and Da-Chaihu-Decoction [180]. Yao et al. investigated the different pharmacological effects of herbs in Ma-huang decoction to elucidate the combination principles of TCM [158]. Zhou et al. investigated the underlying mechanisms of efficacy of herbs for eliminating blood stasis and tonifying Qi by linking the drugs, targets and diseases to obtain compound-target-disease associations for reconstructing the biologically-meaningful networks based on systems pharmacology methods [181]. Zhao et al. built a pharmacological system model of Bufei Jianpi formula by absorption filtering, network targeting, 
Table 2 Selected applications of QSP methods in TCM

\begin{tabular}{|c|c|c|}
\hline TCM & Computational method & Refs. \\
\hline $\begin{array}{l}\text { Acori Tatarinowii Rhizoma and } \\
\text { Curcumae Radix }\end{array}$ & Data mining, pathway enrichment, network analysis & {$[140]$} \\
\hline Erigeron breviscapus & $\begin{array}{l}\text { ADME pharmacokinetic screening, target fishing, protein-protein interaction } \\
\text { network analysis and in vitro experiments verification }\end{array}$ & {$[141]$} \\
\hline Eucommia ulmoides Oliv. & $\begin{array}{l}\text { Drug-likeness evaluation, oral bioavailability prediction, multiple drug targets } \\
\text { prediction and network pharmacology techniques }\end{array}$ & {$[142]$} \\
\hline Hedyotis diffusa Willd. & $\begin{array}{l}\text { Active component gathering, target prediction, related gene collection, gene } \\
\text { enrichment analysis and network analysis }\end{array}$ & {$[143]$} \\
\hline Licorice & $\begin{array}{l}\text { Oral bioavailability screening, drug-likeness evaluation, blood-brain barrier } \\
\text { permeation, target identification and network analysis }\end{array}$ & [111] \\
\hline $\begin{array}{l}\text { Semen strychni and Tripterygium } \\
\text { wilfordii Hook F. }\end{array}$ & Data mining, target prediction, network analysis & {$[144]$} \\
\hline Sinomenium acutum & Pathway, network and function analyses, data mining & {$[145]$} \\
\hline Anti-Thrombosis Drug from TCMs & Data mining, molecular docking, in silico screening & {$[146]$} \\
\hline $\begin{array}{l}\text { Qi-enriching herbs and blood-tonifying } \\
\text { herbs }\end{array}$ & ADME prediction, target fishing and network analysis & {$[147]$} \\
\hline Baihe Dihuang Tang & ADME/T calculation, target prediction, network analysis & {$[148]$} \\
\hline Bufei Jianpi formula & $\begin{array}{l}\text { Systems pharmacology modeling based on absorption filtering, network targeting } \\
\text { and systems analyses }\end{array}$ & {$[132,149]$} \\
\hline Bushenhuoxue formula & Target screening, molecular docking, network analysis, literature mining & {$[150]$} \\
\hline Bushen-Yizhi prescription & ADME/T filter analysis, target prediction, network analysis & {$[151]$} \\
\hline Danlu Capsules & Oral bioavailability and drug-likeness evaluation, gene enrichment analysis & {$[152]$} \\
\hline Danggui-shaoyao-san & $\begin{array}{l}\text { Oral bioavailability screening, drug-likeness assessment, target identification and } \\
\text { network analysis }\end{array}$ & [153] \\
\hline Diesun Miaofang & $\begin{array}{l}\text { Cluster ligands, human intestinal absorption and aqueous solution prediction, } \\
\text { chemical space mapping, molecular docking and network pharmacology techniques }\end{array}$ & {$[154]$} \\
\hline Dragon's blood tablets & Chemical analysis, prediction of ADME, and network analysis & {$[155]$} \\
\hline Ge-Gen-Qin-Lian decoction & Target profile clustering, network target analysis & {$[156]$} \\
\hline Liu-Wei-Di-Huang pill & Chemical and therapeutic properties, network analysis & {$[157]$} \\
\hline Ma-huang decoction & Pharmacokinetic analysis, drug targeting, and drug-target-disease network analysis & {$[158]$} \\
\hline Mahuang Fuzi Xixin decoction & $\begin{array}{l}\text { Drug-likeness evaluation, oral bioavailability prediction, multiple drug target } \\
\text { prediction, and network analysis }\end{array}$ & {$[159]$} \\
\hline MaZiRenWan & $\begin{array}{l}\text { UPLC-QTOF-MS/MS identification, hierarchical clustering analysis, in vitro } \\
\text { experiment verification, network analysis }\end{array}$ & {$[160]$} \\
\hline NiaoDuQing granules & $\begin{array}{l}\text { ADME modelling and target prediction, topology analysis, pathway enrichment } \\
\text { analysis, rat test }\end{array}$ & {$[161]$} \\
\hline Qigui Tongfeng tablet & Molecular similarity analysis, network analysis & {$[162]$} \\
\hline Radix Curcumae formula & $\begin{array}{l}\text { Chemical predictors based on chemical structure and chemogenomics data } \\
\text { linking compounds, pharmacological information, a system biology functional } \\
\text { data analysis and network reconstruction method }\end{array}$ & [163] \\
\hline Reduning injection & $\begin{array}{l}\text { ADME filtering, network targeting, pathways integrating, target selection, reverse } \\
\text { drug targeting and network analysis }\end{array}$ & {$[164-166]$} \\
\hline Shenmai injection & Network construction, network recovery index evaluation & {$[167]$} \\
\hline SiNiSan formula & ADME screening, targets prediction, and DAVID enrichment analysis, & {$[168,169]$} \\
\hline Taohong Siwu decoction & $\begin{array}{l}\text { Chemical space analysis, virtual screening, chemical distribution and potential } \\
\text { compound prediction }\end{array}$ & {$[170]$} \\
\hline Tian-Ma-Gou-Teng-Yin fomula & Network link prediction and statistical analysis & {$[171]$} \\
\hline Tianshu formula & Pharmacokinetic filtering, target fishing and network analysis & {$[172]$} \\
\hline Xiaoyaosan & Reversed pharmacophore matching method, network analysis & {$[173]$} \\
\hline Xijiao Dihuang decoction & ADME screening, drug targeting, network and pathway analysis & [174] \\
\hline
\end{tabular}




\begin{tabular}{llc} 
& & (Continued) \\
\hline TCM & Computational method & Refs. \\
\hline Xin-Sheng-Hua granule & $\begin{array}{l}\text { Plasma metabolomics profiling with UHPLC-QTOF/MS and multivariate data } \\
\text { method, network analysis }\end{array}$ & {$[175]$} \\
Xing-Nao-Jing & $\begin{array}{l}\text { Drug-likeness and brain-blood-barrier evaluation, biological process and } \\
\text { pathway enrichment analyses }\end{array}$ & {$[130]$} \\
Yangxinshi tablet & Molecular docking, network analysis & {$[176]$} \\
Yinchenhao decoction & Oral bioavailability screening, drug-likeness and intestinal epithelial & {$[177]$} \\
& $\begin{array}{l}\text { permeability evaluation, target prediction, pathway identification and network } \\
\text { Zhi-Zi-Da-Huang decoction }\end{array}$ & construction \\
Ginsenoside Rb1, ginsenoside Rg1, \\
schizandrin and DT-13 (effective \\
$\begin{array}{l}\text { compounds from ShengMai } \\
\text { preparations) }\end{array}$
\end{tabular}

and systems analysis and identified 145 bioactive ingredients and 175 potential targets [149]. The model also provides insights of potential synergistic effects between herbs which links with similar targets. Wang et al. used a systems pharmacology method to provide new insights into the pharmacological interactions of Ophiocordyceps sinensis so as to find new adjuvant for hepatitis B vaccine [182]. Yang et al. built an in silico model to predict potential P-Glycoprotein inhibitors and select out 875 potential P-Glycoprotein inhibitors and 15 inhibitorrich herbs from TCMSP [75]. These results make TCM more reasonable and promote the modernization of TCM.

\section{FUTURE PROSPECT}

QSP integrates various types of in vivo and in vitro results from different research areas. QSP methods can simulate a series of biological processes and diseases for multiplescale and systematic exploration of MoA of drugs. The biological responses and changes in disease treatments from the molecular and genetic level to systems level provide a deep insight into these processes. It can also make up quantitative and credible predictions for complex disease while the pathogenesis is not yet fully understood. There are still many challenges in both developing QSP methods and applications. The lack of biological and pharmacological details for complex disease leads to deviations in simulations. Analytical and comprehensive multi-level evaluation methods are urgently needed to construct the appropriate models. Complex associations between factors involved in MoA of drugs further increase the difficulty to obtain meaningful results by analyzing the predictions of modeling. With the development of omics technologies and mathematical techniques such as network dynamics, ordinary differential equations, logic-based approaches, statistical regression and finite element methods, QSP will help to understand the MoA of drugs and TCM, and to improve the efficiency of drug discovery.

\section{ACKNOWLEDGEMENTS}

This work was supported by the start-up support for scientific research of Xinglin Young Scholar in Guangzhou University of Chinese Medicine (A1AFD018161Z04).

\section{COMPLIANCE WITH ETHICAL GUIDELINES}

Fuda Xie and Jiangyong Gu declare that they have no conflict of interests.

This article is a review article and does not contain any studies with human or animal subjects performed by any of the authors.

\section{REFERENCES}

1. Berger, S. I. and Iyengar, R. (2009) Network analyses in systems pharmacology. Bioinformatics, 25, 2466-2472

2. Zhao, S. and Iyengar, R. (2012) Systems pharmacology: network analysis to identify multiscale mechanisms of drug action. Annu. Rev. Pharmacol. Toxicol., 52, 505-521

3. Boran, A. D. and Iyengar, R. (2010) Systems pharmacology. Mt. Sinai J. Med., 77, 333-344

4. Zhou, W., Wang, Y., Lu, A. and Zhang, G. (2016) Systems pharmacology in small molecular drug discovery. Int. J. Mol. Sci., 17,246

5. Gu, J., Zhang, X., Ma, Y., Li, N., Luo, F., Cao, L., Wang, Z., Yuan, G., Chen, L., Xiao, W., et al. (2015) Quantitative modeling of dose-response and drug combination based on pathway network. J. Cheminform., 7, 19

6. Spiros, A., Roberts, P. and Geerts, H. (2014) A computer-based quantitative systems pharmacology model of negative symptoms in schizophrenia: exploring glycine modulation of excitationinhibition balance. Front. Pharmacol., 5, 229

7. Fang, J., Wu, Z., Cai, C., Wang, Q., Tang, Y. and Cheng, F. (2017) Quantitative and systems pharmacology. 1. in silico prediction of drug-target interactions of natural products enables new targeted 
cancer therapy. J. Chem. Inf. Model., 57, 2657-2671

8. Fleisher, B., Brown, A. N. and Ait-Oudhia, S. (2017) Application of pharmacometrics and quantitative systems pharmacology to cancer therapy: the example of luminal a breast cancer. Pharmacol. Res., 124, 20-33

9. Geerts, H., Spiros, A. and Roberts, P. (2018) Impact of amyloidbeta changes on cognitive outcomes in Alzheimer's disease: analysis of clinical trials using a quantitative systems pharmacology model. Alzheimers Res. Ther., 10, 14

10. Barabási, A. L., Gulbahce, N. and Loscalzo, J. (2011) Network medicine: a network-based approach to human disease. Nat. Rev. Genet., 12, 56-68

11. Pérez-Nueno, V. I. (2015) Using quantitative systems pharmacology for novel drug discovery. Expert Opin. Drug Discov., 10, 1315-1331

12. Woodhead, J. L., Watkins, P. B., Howell, B. A., Siler, S. Q. and Shoda, L. K. M. (2017) The role of quantitative systems pharmacology modeling in the prediction and explanation of idiosyncratic drug-induced liver injury. Drug Metab. Pharmacokinet., 32, 40-45

13. Androulakis, I. P. (2016) Quantitative systems pharmacology: a framework for context. Curr. Pharmacol. Rep., 2, 152-160

14. van der Graaf, P. H. and Benson, N. (2011) Systems pharmacology: bridging systems biology and pharmacokineticspharmacodynamics (PKPD) in drug discovery and development. Pharm. Res., 28, 1460-1464

15. Leil, T. A. and Bertz, R. (2014) Quantitative systems pharmacology can reduce attrition and improve productivity in pharmaceutical research and development. Front. Pharmacol., 5, 247

16. Rao, R. T., Scherholz, M. L., Hartmanshenn, C., Bae, S. A. and Androulakis, I. P. (2017) On the analysis of complex biological supply chains: from process systems engineering to quantitative systems pharmacology. Comput. Chem. Eng., 107, 100-110

17. Yu, J., Cilfone, N. A., Large, E. M., Sarkar, U., Wishnok, J. S., Tannenbaum, S. R., Hughes, D. J., Lauffenburger, D. A., Griffith, L. G., Stokes, C. L., et al. (2015) Quantitative systems pharmacology approaches applied to microphysiological systems (MPS): data interpretation and multi-MPS integration. CPT Pharmacometrics Syst. Pharmacol., 4, 585-594

18. Musante, C. J., Abernethy, D. R., Allerheiligen, S. R., Lauffenburger, D. A. and Zager, M. G. (2016) GPS for QSP: A summary of the ACoP6 symposium on quantitative systems pharmacology and a stage for near-term efforts in the field. CPT Pharmacometrics Syst. Pharmacol., 5, 449-451

19. Ribba, B., Grimm, H. P., Agoram, B., Davies, M. R., Gadkar, K., Niederer, S., van Riel, N., Timmis, J. and van der Graaf, P. H. (2017) Methodologies for quantitative systems pharmacology (QSP) models: design and estimation. CPT Pharmacometrics Syst. Pharmacol., 6, 496-498

20. Timmis, J., Alden, K., Andrews, P., Clark, E., Nellis, A., Naylor, B., Coles, M. and Kaye, P. (2017) Building confidence in quantitative systems pharmacology models: an engineer's guide to exploring the rationale in model design and development. CPT
Pharmacometrics Syst. Pharmacol., 6, 156-167

21. Cherkaoui-Rbati, M. H., Paine, S. W., Littlewood, P. and Rauch, C. (2017) A quantitative systems pharmacology approach, incorporating a novel liver model, for predicting pharmacokinetic drug-drug interactions. PLoS One, 12, e0183794

22. Rogers, M., Lyster, P. and Okita, R. (2013) NIH support for the emergence of quantitative and systems pharmacology. CPT Pharmacometrics Syst. Pharmacol., 2, e37

23. Wist, A. D., Berger, S. I. and Iyengar, R. (2009) Systems pharmacology and genome medicine: a future perspective. Genome Med., 1, 11

24. Wang, Z. and Deisboeck, T. S. (2014) Mathematical modeling in cancer drug discovery. Drug Discov. Today, 19, 145-150

25. Medina-Franco, J. L., Giulianotti, M. A., Welmaker, G. S. and Houghten, R. A. (2013) Shifting from the single to the multitarget paradigm in drug discovery. Drug Discov. Today, 18, 495-501

26. Hopkins, A. L. (2007) Network pharmacology. Nat. Biotechnol., $25,1110-1111$

27. Goh, K. I. and Choi, I. G. (2012) Exploring the human diseasome: the human disease network. Brief. Funct. Genomics, 11, 533-542

28. Goh, K. I., Cusick, M. E., Valle, D., Childs, B., Vidal, M. and Barabási, A. L. (2007) The human disease network. Proc. Natl. Acad. Sci. USA, 104, 8685-8690

29. Zhang, W., Pei, J. and Lai, L. (2017) Computational multitarget drug design. J. Chem. Inf. Model., 57, 403-412

30. Yildirim, M. A., Goh, K. I., Cusick, M. E., Barabási, A. L. and Vidal, M. (2007) Drug-target network. Nat. Biotechnol., 25, 1119-1126

31. Barneh, F., Jafari, M. and Mirzaie, M. (2016) Updates on drugtarget network; facilitating polypharmacology and data integration by growth of DrugBank database. Brief. Bioinformatics, 17, 1070-1080

32. Geerts, H., Spiros, A., Roberts, P. and Carr, R. (2013) Quantitative systems pharmacology as an extension of PK/PD modeling in CNS research and development. J. Pharmacokinet. Pharmacodyn., 40, 257-265

33. Snelder, N., Ploeger, B. A., Luttringer, O., Rigel, D. F., Fu, F., Beil, M., Stanski, D. R. and Danhof, M. (2014) Drug effects on the CVS in conscious rats: separating cardiac output into heart rate and stroke volume using PKPD modelling. Br. J. Pharmacol., 171, 5076-5092

34. Hansson, E. K., Amantea, M. A., Westwood, P., Milligan, P. A., Houk, B. E., French, J., Karlsson, M. O. and Friberg, L. E. (2013) PKPD Modeling of VEGF, sVEGFR-2, sVEGFR-3, and sKIT as predictors of tumor dynamics and overall survival following sunitinib treatment in GIST. CPT Pharmacometrics Syst. Pharmacol., 2, e84

35. Reymond, J. L. and Awale, M. (2012) Exploring chemical space for drug discovery using the chemical universe database. ACS Chem. Neurosci., 3, 649-657

36. Tian, S., Wang, J., Li, Y., Li, D., Xu, L. and Hou, T. (2015) The application of in silico drug-likeness predictions in pharmaceutical research. Adv. Drug Deliv. Rev., 86, 2-10

37. May, E. R. (2014) Recent developments in molecular simulation 
approaches to study spherical virus capsids. Mol. Simul., 40, 878-888

38. Field, M. J. (2015) Technical advances in molecular simulation since the 1980s. Arch. Biochem. Biophys., 582, 3-9

39. Xie, L., Draizen, E. J. and Bourne, P. E. (2017) Harnessing big data for systems pharmacology. Annu. Rev. Pharmacol. Toxicol., 57, 245-262

40. Liu, X., Zhu, F., Ma, X. H., Shi, Z., Yang, S. Y., Wei, Y. Q. and Chen, Y. Z. (2013) Predicting targeted polypharmacology for drug repositioning and multi- target drug discovery. Curr. Med. Chem., 20, 1646-1661

41. Chiu, S. H. and Xie, L. (2016) Toward high-throughput predictive modeling of protein binding/unbinding kinetics. J. Chem. Inf. Model., 56, 1164-1174

42. Hart, T. and Xie, L. (2016) Providing data science support for systems pharmacology and its implications to drug discovery. Expert Opin. Drug Discov., 11, 241-256

43. Bloomingdale, P., Nguyen, V. A., Niu, J. and Mager, D. E. (2018) Boolean network modeling in systems pharmacology. J. Pharmacokinet. Pharmacodyn., 45, 159-180

44. Irurzun-Arana, I., Pastor, J. M., Trocóniz, I. F. and GómezMantilla, J. D. (2017) Advanced Boolean modeling of biological networks applied to systems pharmacology. Bioinformatics, 33, 1040-1048

45. Danhof, M. (2016) Systems pharmacology — towards the modeling of network interactions. Eur. J. Pharm. Sci., 94, 4-14

46. Tang, Y., Tang, Q., Dong, C., Li, X., Zhang, Z. and An, F. (2015) Protein-protein interaction network and mechanism analysis of hepatitis C. Genet. Mol. Res., 14, 2069-2079

47. Schurdak, M. E., Pei, F., Lezon, T. R., Carlisle, D., Friedlander, R., Taylor, D. L. and Stern, A. M. (2018) A quantitative systems pharmacology approach to infer pathways involved in complex disease phenotypes. Methods Mol. Biol., 1787, 207-222

48. Li, Q., Li, X., Li, C., Chen, L., Song, J., Tang, Y. and Xu, X. (2011) A network-based multi-target computational estimation scheme for anticoagulant activities of compounds. PLoS One, 6, e14774

49. Zhang, X., Gu, J., Cao, L., Ma, Y., Su, Z., Luo, F., Wang, Z., Li, N., Yuan, G., Chen, L., et al. (2014) Insights into the inhibition and mechanism of compounds against LPS-induced PGE2 production: a pathway network-based approach and molecular dynamics simulations. Integr. Biol., 6, 1162-1169

50. Gu, J., Li, Q., Chen, L., Li, Y., Hou, T., Yuan, G. and Xu, X. (2013) Platelet aggregation pathway network-based approach for evaluating compounds efficacy. Evid. Based Complement. Alternat. Med., 2013, 425707

51. Traynard, P., Tobalina, L., Eduati, F., Calzone, L. and Saez-Rodriguez, J. (2017) Logic modeling in quantitative systems pharmacology. CPT Pharmacometrics Syst. Pharmacol., 6, 499-511

52. Ru, J., Li, P., Wang, J., Zhou, W., Li, B., Huang, C., Li, P., Guo, Z., Tao, W., Yang, Y., et al. (2014) TCMSP: a database of systems pharmacology for drug discovery from herbal medicines. J. Cheminform., 6, 13
53. Chassagnole, C., Jackson, R. C., Hussain, N., Bashir, L., Derow, C., Savin, J. and Fell, D. A. (2006) Using a mammalian cell cycle simulation to interpret differential kinase inhibition in anti-tumour pharmaceutical development. Biosystems, 83, 91-97

54. Tao, W., Li, B., Gao, S., Bai, Y., Shar, P. A., Zhang, W., Guo, Z., Sun, K., Fu, Y., Huang, C., et al. (2015) CancerHSP: anticancer herbs database of systems pharmacology. Sci. Rep., 5, 11481

55. Huang, H., Wu, X., Pandey, R., Li, J., Zhao, G., Ibrahim, S. and Chen, J. Y. (2012) $C^{2}$ Maps: a network pharmacology database with comprehensive disease-gene-drug connectivity relationships. BMC Genomics, 13, S17

56. Hu, Z., Chang, Y. C., Wang, Y., Huang, C. L., Liu, Y., Tian, F., Granger, B. and Delisi, C. (2013) VisANT 4.0: integrative network platform to connect genes, drugs, diseases and therapies. Nucleic Acids Res., 41, W225-W 231

57. Wang, D., Gu, J., Zhu, W., Luo, F., Chen, L., Xu, X. and Lu, C. (2017) PDTCM: a systems pharmacology platform of traditional Chinese medicine for psoriasis. Ann. Med., 49, 652-660

58. Gu, J., Gui, Y., Chen, L., Yuan, G. and Xu, X. (2013) CVDHD: a cardiovascular disease herbal database for drug discovery and network pharmacology. J. Cheminform., 5, 51

59. Musante, C. J., Ramanujan, S., Schmidt, B. J., Ghobrial, O. G., Lu, J. and Heatherington, A. C. (2017) Quantitative systems pharmacology: a case for disease models. Clin. Pharmacol. Ther., 101, 24-27

60. Schmidt, B. J., Casey, F. P., Paterson, T. and Chan, J. R. (2013) Alternate virtual populations elucidate the type I interferon signature predictive of the response to rituximab in rheumatoid arthritis. BMC Bioinformatics, 14, 221

61. Ghosh, S., Matsuoka, Y., Asai, Y., Hsin, K. Y. and Kitano, H. (2013) Toward an integrated software platform for systems pharmacology. Biopharm. Drug Dispos., 34, 508-526

62. Spiros, A., Roberts, P. and Geerts, H. (2013) Phenotypic screening of the Prestwick library for treatment of Parkinson's tremor symptoms using a humanized quantitative systems pharmacology platform. J Parkinsons Dis, 3, 569-580

63. Ming, J. E., Abrams, R. E., Bartlett, D. W., Tao, M., Nguyen, T., Surks, H., Kudrycki, K., Kadambi, A., Friedrich, C. M., Djebli, N., et al. (2017) A quantitative systems pharmacology platform to investigate the impact of alirocumab and cholesterol-lowering therapies on lipid profiles and plaque characteristics. Gene Regul. Syst. Bio., 11, 1177625017710941

64. Zheng, C., Pei, T., Huang, C., Chen, X., Bai, Y., Xue, J., Wu, Z., $\mathrm{Mu}$, J., Li, Y. and Wang, Y. (2016) A novel systems pharmacology platform to dissect action mechanisms of traditional Chinese medicines for bovine viral diarrhea disease. Eur. J. Pharm. Sci., 94, 33-45

65. Rieger, T. R., Allen, R. J., Bystricky, L., Chen, Y., Colopy, G. W., Cui, Y., Gonzalez, A., Liu, Y., White, R. D., Everett, R. A., et al. (2018) Improving the generation and selection of virtual populations in quantitative systems pharmacology models. Prog. Biophys. Mol. Biol., 139, 15-22

66. Geerts, H., Spiros, A., Roberts, P. and Carr, R. (2017) Towards the virtual human patient. quantitative systems pharmacology in 
Alzheimer's disease. Eur. J. Pharmacol., 817, 38-45

67. Wiśniowska, B. and Polak, S. (2016) Virtual clinical trial toward polytherapy safety assessment: combination of physiologically based pharmacokinetic/pharmacodynamic-based modeling and simulation approach with drug-drug interactions involving terfenadine as an example. J. Pharm. Sci., 105, 3415-3424

68. Allen, R. J., Rieger, T. R. and Musante, C. J. (2016) Efficient generation and selection of virtual populations in quantitative systems pharmacology models. CPT Pharmacometrics Syst. Pharmacol., 5, 140-146

69. Rostami-Hodjegan, A. (2012) Physiologically based pharmacokinetics joined with in vitro-in vivo extrapolation of ADME: a marriage under the arch of systems pharmacology. Clin. Pharmacol. Ther., 92, 50-61

70. Bloomingdale, P., Housand, C., Apgar, J. F., Millard, B. L., Mager, D. E., Burke, J. M. and Shah, D. K. (2017) Quantitative systems toxicology. Curr. Opin. Toxicol., 4, 79-87

71. Pichardo-Almarza, C. and Diaz-Zuccarini, V. (2017) From PK/ PD to QSP: understanding the dynamic effect of cholesterollowering drugs on atherosclerosis progression and stratified medicine. Curr. Pharm. Des., 22, 6903-6910

72. Meng, X. Y., Zhang, H. X., Mezei, M. and Cui, M. (2011) Molecular docking: a powerful approach for structure-based drug discovery. Curr. Comput. Aided Drug Des., 7, 146-157

73. Omer, A. and Singh, P. (2017) An integrated approach of network-based systems biology, molecular docking, and molecular dynamics approach to unravel the role of existing antiviral molecules against AIDS-associated cancer. J. Biomol. Struct. Dyn., 35, 1547-1558

74. Gu, J., Li, L., Wang, D., Zhu, W., Han, L., Zhao, R., Xu, X. and $\mathrm{Lu}$, C. (2018) Deciphering metabonomics biomarkers-targets interactions for psoriasis vulgaris by network pharmacology. Ann. Med., 50, 323-332

75. Yang, M., Chen, J., Shi, X., Xu, L., Xi, Z., You, L., An, R. and Wang, X. (2015) Development of in silico models for predicting p-glycoprotein inhibitors based on a two-step approach for feature selection and its application to Chinese herbal medicine screening. Mol. Pharm., 12, 3691-3713

76. Gilson, M. K., Liu, T., Baitaluk, M., Nicola, G., Hwang, L. and Chong, J. (2016) BindingDB in 2015: a public database for medicinal chemistry, computational chemistry and systems pharmacology. Nucleic Acids Res., 44, D1045-D1053

77. Liu, Z., Guo, F., Wang, Y., Li, C., Zhang, X., Li, H., Diao, L., Gu, J., Wang, W., Li, D., et al. (2016) BATMAN-TCM: a bioinformatics analysis Tool for molecular mechanism of traditional Chinese medicine. Sci. Rep., 6, 21146

78. Boran, A. D. and Iyengar, R. (2010) Systems approaches to polypharmacology and drug discovery. Curr Opin Drug Discov Devel, 13, 297-309

79. Berger, S. I., Ma'ayan, A. and Iyengar, R. (2010) Systems pharmacology of arrhythmias. Sci. Signal., 3, ra30

80. Boland, M. R., Jacunski, A., Lorberbaum, T., Romano, J. D., Moskovitch, R. and Tatonetti, N. P. (2016) Systems biology approaches for identifying adverse drug reactions and elucidating their underlying biological mechanisms. Wiley Interdiscip. Rev. Syst. Biol. Med., 8, 104-122

81. Goldstein, L. H., Berlin, M., Saliba, W., Elias, M. and Berkovitch, M. (2013) Founding an adverse drug reaction (ADR) network: a method for improving doctors spontaneous ADR reporting in a general hospital. J. Clin. Pharmacol., 53, 1220-1225

82. Zhao, S., Nishimura, T., Chen, Y., Azeloglu, E. U., Gottesman, O., Giannarelli, C., Zafar, M. U., Benard, L., Badimon, J. J., Hajjar, R. J., et al. (2013) Systems pharmacology of adverse event mitigation by drug combinations. Sci. Transl. Med., 5, 206ra140

83. Wu, Z., Cheng, F., Li, J., Li, W., Liu, G. and Tang, Y. (2017) SDTNBI: an integrated network and chemoinformatics tool for systematic prediction of drug-target interactions and drug repositioning. Brief. Bioinformatics, 18, 333-347

84. Wu, Z., Lu, W., Wu, D., Luo, A., Bian, H., Li, J., Li, W., Liu, G., Huang, J., Cheng, F., et al. (2016) In silico prediction of chemical mechanism of action via an improved network-based inference method. Br. J. Pharmacol., 173, 3372-3385

85. Wang, J., Guo, Z., Fu, Y., Wu, Z., Huang, C., Zheng, C., Shar, P. A., Wang, Z., Xiao, W. and Wang, Y. (2017) Weak-binding molecules are not drugs? - toward a systematic strategy for finding effective weak-binding drugs. Brief. Bioinformatics, 18, 321-332

86. Huang, C., Zheng, C., Li, Y., Wang, Y., Lu, A. and Yang, L. (2014) Systems pharmacology in drug discovery and therapeutic insight for herbal medicines. Brief. Bioinform., 15, 710-733

87. Mitrea, C., Taghavi, Z., Bokanizad, B., Hanoudi, S., Tagett, R., Donato, M., Voichița, C. and Drăghici, S. (2013) Methods and approaches in the topology-based analysis of biological pathways. Front. Physiol., 4, 278

88. Nie, X. Z., Du, X., Zhang, R. R., He, J., Su, R., Ma, H. Q., Mu, J., Li, Y. and Liu, F. (2017) Study on regulation mechanism of Toutongning capsule through TNF signaling pathway in treatment of migraine based on systems pharmacology method. Zhongguo Zhongyao Zazhi, 42, 548-554, in Chinese

89. Gu, J., Crosier, P. S., Hall, C. J., Chen, L. and Xu, X. (2016) Inflammatory pathway network-based drug repositioning and molecular phenomics. Mol. Biosyst., 12, 2777-2784

90. Poltz, R. and Naumann, M. (2012) Dynamics of p53 and NF- $\kappa B$ regulation in response to DNA damage and identification of target proteins suitable for therapeutic intervention. BMC Syst. Biol., 6, 125

91. Le Novère, N. (2015) Quantitative and logic modelling of molecular and gene networks. Nat. Rev. Genet., 16, 146-158

92. Chaouiya, C. and Remy, E. (2013) Logical modelling of regulatory networks, methods and applications. Bull. Math. Biol., 75, 891-895

93. Kirouac, D. C., Du, J. Y., Lahdenranta, J., Overland, R., Yarar, D., Paragas, V., Pace, E., McDonagh, C. F., Nielsen, U. B. and Onsum, M. D. (2013) Computational modeling of ERBB2amplified breast cancer identifies combined ErbB2/3 blockade as superior to the combination of MEK and AKT inhibitors. Sci. Signal., 6, ra68

94. Shoda, L. K., Woodhead, J. L., Siler, S. Q., Watkins, P. B. and 
Howell, B. A. (2014) Linking physiology to toxicity using DILIsym ${ }^{\circledR}$, a mechanistic mathematical model of drug-induced liver injury. Biopharm. Drug Dispos., 35, 33-49

95. Woodhead, J. L., Yang, K., Siler, S. Q., Watkins, P. B., Brouwer, K. L., Barton, H. A. and Howell, B. A. (2014) Exploring BSEP inhibition-mediated toxicity with a mechanistic model of druginduced liver injury. Front. Pharmacol., 5, 240

96. Woodhead, J. L., Paech, F., Maurer, M., Engelhardt, M., SchmittHoffmann, A. H., Spickermann, J., Messner, S., Wind, M., Witschi, A. T., Krähenbühl, S., et al. (2018) Prediction of safety margin and optimization of dosing protocol for a novel antibiotic using quantitative systems pharmacology modeling. Clin. Transl. Sci., 11, 498-505

97. Geerts, H., Roberts, P. and Spiros, A. (2015) Assessing the synergy between cholinomimetics and memantine as augmentation therapy in cognitive impairment in schizophrenia. A virtual human patient trial using quantitative systems pharmacology. Front. Pharmacol., 6, 198

98. Hopkins, A. L. (2008) Network pharmacology: the next paradigm in drug discovery. Nat. Chem. Biol., 4, 682-690

99. Allerheiligen, S. R. (2010) Next-generation model-based drug discovery and development: quantitative and systems pharmacology. Clin. Pharmacol. Ther., 88, 135-137

100. Agoram, B. M. and Demin, O. (2011) Integration not isolation: arguing the case for quantitative and systems pharmacology in drug discovery and development. Drug Discov. Today, 16, 10311036

101. Geerts, H. and Kennis, L. (2014) Multitarget drug discovery projects in CNS diseases: quantitative systems pharmacology as a possible path forward. Future Med. Chem., 6, 1757-1769

102. Fang, J., Gao, L., Ma, H., Wu, Q., Wu, T., Wu, J., Wang, Q. and Cheng, F. (2017) Quantitative and systems pharmacology 3. network-based identification of new targets for natural products enables potential uses in aging-associated disorders. Front. Pharmacol., 8, 747

103. Janga, S. C. and Tzakos, A. (2009) Structure and organization of drug-target networks: insights from genomic approaches for drug discovery. Mol. Biosyst., 5, 1536-1548

104. Arrell, D. K. and Terzic, A. (2010) Network systems biology for drug discovery. Clin. Pharmacol. Ther., 88, 120-125

105. Knight-Schrijver, V. R., Chelliah, V., Cucurull-Sanchez, L. and Le Novère, N. (2016) The promises of quantitative systems pharmacology modelling for drug development. Comput. Struct. Biotechnol. J., 14, 363-370.

106. Kim, S., Lahu, G., Lesko, L. J. and Trame, M. N. (2017) An exemplar of a systems pharmacology approach for a detailed investigation of an adverse drug event as a result of drug-drug interactions. Clin. Pharmacol. Ther., 101, S97-S97.

107. Kariya, Y., Honma, M. and Suzuki, H. (2016) Mechanism analyses and mechanism-based prediction for adverse drug reactions using systems pharmacology. Nippon Yakurigaku Zasshi, 147, 89-94, in Japanese

108. Cao, D. S., Xiao, N., Li, Y. J., Zeng, W. B., Liang, Y. Z., Lu, A. P., Xu, Q. S. and Chen, A. F. (2015) Integrating multiple evidence sources to predict adverse drug reactions based on a systems pharmacology model. CPT Pharmacometrics Syst. Pharmacol., 4, 498-506

109. Berger, S. I. and Iyengar, R. (2011) Role of systems pharmacology in understanding drug adverse events. Wiley Interdiscip. Rev. Syst. Biol. Med., 3, 129-135

110. Nueno, V. I. (2016) Towards the integration of quantitative and systems pharmacology into drug discovery: a systems level understanding of therapeutic and toxic effects of drugs. Curr. Pharm. Des., 22, 6881-6884

111. Liu, H., Wang, J., Zhou, W., Wang, Y. and Yang, L. (2013) Systems approaches and polypharmacology for drug discovery from herbal medicines: an example using licorice. J. Ethnopharmacol., 146, 773-793

112. Luo, F., Gu, J., Chen, L. and Xu, X. (2014) Systems pharmacology strategies for anticancer drug discovery based on natural products. Mol. Biosyst., 10, 1912-1917

113. Dziuba, J., Alperin, P., Racketa, J., Iloeje, U., Goswami, D., Hardy, E., Perlstein, I., Grossman, H. L. and Cohen, M. (2014) Modeling effects of SGLT-2 inhibitor dapagliflozin treatment versus standard diabetes therapy on cardiovascular and microvascular outcomes. Diabetes Obes. Metab., 16, 628-635

114. Peskin, B. R., Shcheprov, A. V., Boye, K. S., Bruce, S., Maggs, D. G. and Gaebler, J. A. (2011) Cardiovascular outcomes associated with a new once-weekly GLP-1 receptor agonist vs. traditional therapies for type 2 diabetes: a simulation analysis. Diabetes Obes. Metab., 13, 921-927

115. Gadkar, K., Kirouac, D., Parrott, N. and Ramanujan, S. (2016) Quantitative systems pharmacology: a promising approach for translational pharmacology. Drug Discov. Today. Technol., 2122, 57-65

116. Cirit, M. and Stokes, C. L. (2018) Maximizing the impact of microphysiological systems with in vitro-in vivo translation. Lab Chip, 18, 1831-1837

117. Yuraszeck, T., Kasichayanula, S. and Benjamin, J. E. (2017) Translation and clinical development of bispecific T-cell engaging antibodies for cancer treatment. Clin. Pharmacol. Ther., 101, 634645

118. Schulthess, P., Post, T. M., Yates, J. and van der Graaf, P. H. (2018) Frequency-domain response analysis for quantitative systems pharmacology models. CPT Pharmacometrics Syst. Pharmacol.,7,111-123

119. Visser, S. A., de Alwis, D. P., Kerbusch, T., Stone, J. A. and Allerheiligen, S. R. (2014) Implementation of quantitative and systems pharmacology in large pharma. CPT Pharmacometrics Syst. Pharmacol., 3, e142

120. Geerts, H., Roberts, P. and Spiros, A. (2013) A quantitative system pharmacology computer model for cognitive deficits in schizophrenia. CPT Pharmacometrics Syst. Pharmacol., 2, e36

121. Liu, J., Ogden, A., Comery, T. A., Spiros, A., Roberts, P. and Geerts, H. (2014) Prediction of efficacy of vabicaserin, a 5-HT2C agonist, for the treatment of schizophrenia using a quantitative systems pharmacology model. CPT Pharmacometrics Syst. Pharmacol., 3, e111 
122. Geerts, H., Roberts, P., Spiros, A. and Potkin, S. (2015) Understanding responder neurobiology in schizophrenia using a quantitative systems pharmacology model: application to iloperidone. J. Psychopharmacol. (Oxford), 29, 372-382

123. Vega-Villa, K., Pluta, R., Lonser, R. and Woo, S. (2013) Quantitative systems pharmacology model of NO metabolome and methemoglobin following long-term infusion of sodium nitrite in humans. CPT Pharmacometrics Syst. Pharmacol., 2, e60

124. John, T., Kiss, T., Lever, C. and Érdi, P. (2014) Anxiolytic drugs and altered hippocampal theta rhythms: the quantitative systems pharmacological approach. Network, 25, 20-37

125. Johnson, T. N. and Rostami-Hodjegan, A. (2011) Resurgence in the use of physiologically based pharmacokinetic models in pediatric clinical pharmacology: parallel shift in incorporating the knowledge of biological elements and increased applicability to drug development and clinical practice. Paediatr. Anaesth., 21, 291-301

126. Kaddi, C. D., Niesner, B., Baek, R., Jasper, P., Pappas, J., Tolsma, J., Li, J., van Rijn, Z., Tao, M., Ortemann-Renon, C., et al. (2018) Quantitative systems pharmacology modeling of acid sphingomyelinase deficiency and the enzyme replacement therapy olipudase alfa is an innovative tool for linking pathophysiology and pharmacology. CPT Pharmacometrics Syst. Pharmacol., 7, $442-452$

127. Stern, A. M., Schurdak, M. E., Bahar, I., Berg, J. M. and Taylor, D. L. (2016) A perspective on implementing a quantitative systems pharmacology platform for drug discovery and the advancement of personalized medicine. J. Biomol. Screen., 21, 521-534

128. Geerts, H., Spiros, A., Roberts, P. and Alphs, L. (2018) A quantitative systems pharmacology study on optimal scenarios for switching to paliperidone palmitate once-monthly. Schizophr. Res., 197, 261-268

129. Yin, A., Yamada, A., Stam, W. B., van Hasselt, J. G. C. and van der Graaf, P. H. (2018) Quantitative systems pharmacology analysis of drug combination and scaling to humans: the interaction between noradrenaline and vasopressin in vasoconstriction. Br. J. Pharmacol., 175, 3394-3406

130. Chen, Y., Sun, Y., Li, W., Wei, H., Long, T., Li, H., Xu, Q. and Liu, W. (2018) Systems pharmacology dissection of the antistroke mechanism for the Chinese traditional medicine Xing-NaoJing. J. Pharmacol. Sci., 136, 16-25

131. Li, J., Zhao, P., Li, Y., Tian, Y. and Wang, Y. (2015) Systems pharmacology-based dissection of mechanisms of Chinese medicinal formula Bufei Yishen as an effective treatment for chronic obstructive pulmonary disease. Sci. Rep., 5, 15290

132. Zhao, P., Yang, L., Li, J., Li, Y., Tian, Y. and Li, S. (2016) Combining systems pharmacology, transcriptomics, proteomics, and metabolomics to dissect the therapeutic mechanism of Chinese herbal Bufei Jianpi formula for application to COPD. Int. J. Chron. Obstruct. Pulmon. Dis., 11, 553-566

133. Zhao, P., Li, J., Yang, L., Li, Y., Tian, Y. and Li, S. (2018) Integration of transcriptomics, proteomics, metabolomics and systems pharmacology data to reveal the therapeutic mechanism underlying Chinese herbal Bufei Yishen formula for the treatment of chronic obstructive pulmonary disease. Mol. Med. Rep., 17, 5247-5257

134. Zhang, W., Tao, Q., Guo, Z., Fu, Y., Chen, X., Shar, P. A., Shahen, M., Zhu, J., Xue, J., Bai, Y., et al. (2016) Systems pharmacology dissection of the integrated treatment for cardiovascular and gastrointestinal disorders by traditional Chinese medicine. Sci. Rep., 6, 32400

135. Sun, M., Chang, W. T., Van Wijk, E., He, M., Koval, S., Lin, M. K., Van Wijk, R., Hankemeier, T., van der Greef, J. and Wang, M. (2017) Characterization of the therapeutic properties of Chinese herbal materials by measuring delayed luminescence and dendritic cell-based immunomodulatory response. J. Photochem. Photobiol. B, 168, 1-11

136. Wang, J., Li, Y., Yang, Y., Chen, X., Du, J., Zheng, Q., Liang, Z. and Wang, Y. (2017) A new strategy for deleting animal drugs from traditional Chinese medicines based on modified yimusake formula. Sci. Rep., 7, 1504

137. Ai, H., Wu, X., Qi, M., Zhang, L., Hu, H., Zhao, Q., Zhao, J. and Liu, H. (2018) Study on the mechanisms of active compounds in traditional Chinese medicine for the treatment of influenza virus by virtual screening. Interdiscip. Sci., 10, 320-328

138. Jiang, Q. Y., Zheng, M. S., Yang, X. J. and Sun, X. S. (2016) Analysis of molecular networks and targets mining of Chinese herbal medicines on anti-aging. BMC Complement. Altern. Med., 16,520

139. Liu, J., Liu, J., Shen, F., Qin, Z., Jiang, M., Zhu, J., Wang, Z., Zhou, J., Fu, Y., Chen, X., et al. (2018) Systems pharmacology analysis of synergy of TCM: an example using saffron formula. Sci. Rep., 8, 380

140. Fan, W. T. and Wang, Q. (2018) Mechanism of Acori Tatarinowii Rhizoma-Curcumae Radix treating depression based on network pharmacology. Zhongguo Zhongyao Zazhi, 43, 2607-2611, in Chinese

141. Wang, J., Zhang, L., Liu, B., Wang, Q., Chen, Y., Wang, Z., Zhou, J., Xiao, W., Zheng, C. and Wang, Y. (2018) Systematic investigation of the Erigeron breviscapus mechanism for treating cerebrovascular disease. J. Ethnopharmacol., 224, 429-440

142. Li, Y., Han, C., Wang, J., Xiao, W., Wang, Z., Zhang, J., Yang, Y., Zhang, S. and Ai, C. (2014) Investigation into the mechanism of Eucommia ulmoides Oliv. based on a systems pharmacology approach. J. Ethnopharmacol., 151, 452-460

143. Liu, X., Wu, J., Zhang, D., Wang, K., Duan, X. and Zhang, X. (2018) A network pharmacology approach to uncover the multiple mechanisms of Hedyotis diffusa Willd. on colorectal cancer. Evid. Based Complement. Alternat. Med., 2018, 6517034

144. Li, Y., Wang, J., Xiao, Y., Wang, Y., Chen, S., Yang, Y., Lu, A. and Zhang, S. (2015) A systems pharmacology approach to investigate the mechanisms of action of semen strychni and Tripterygium wilfordii Hook $\mathrm{F}$ for treatment of rheumatoid arthritis. J. Ethnopharmacol., 175, 301-314

145. Li, Y. Y., Zheng, G. and Liu, L. (2018) Bioinformatics based therapeutic effects of Sinomenium Acutum. Chin. J. Integr. Med., 10.1007/s11655-018-2796-6 
146. Yi, F., Sun, L., Xu, L. J., Peng, Y., Liu, H. B., He, C. N. and Xiao, P. G. (2017) In silico approach for anti-thrombosis drug discovery: $\mathrm{P} 2 \mathrm{Y}_{1} \mathrm{R}$ structure-based TCMs screening. Front. Pharmacol., 7, 531

147. Liu, J., Pei, M., Zheng, C., Li, Y., Wang, Y., Lu, A. and Yang, L. (2013) A systems-pharmacology analysis of herbal medicines used in health improvement treatment: predicting potential new drugs and targets. Evid. Based Complement. Alternat. Med., 2013, 938764

148. Zhao, L., Wu, Y. F., Gao, Y., Xiang, H., Qin, X. M. and Tian, J. S. (2017) Intervention mechanism of psychological sub-health by Baihe Dihuang Tang based on network pharmacology. Acta Pharma. Sinica (Yao Xue Xue Bao ), 52, 99-105, in Chinese

149. Zhao, P., Li, J., Li, Y., Tian, Y., Wang, Y. and Zheng, C. (2015) Systems pharmacology-based approach for dissecting the active ingredients and potential targets of the Chinese herbal Bufei Jianpi formula for the treatment of COPD. Int. J. Chron. Obstruct. Pulmon. Dis., 10, 2633-2656

150. Shi, S. H., Cai, Y. P., Cai, X. J., Zheng, X. Y., Cao, D. S., Ye, F. Q. and Xiang, Z. (2014) A network pharmacology approach to understanding the mechanisms of action of traditional medicine: Bushenhuoxue formula for treatment of chronic kidney disease. PLoS One, 9, e89123

151. Cai, H., Luo, Y., Yan, X., Ding, P., Huang, Y., Fang, S., Zhang, R., Chen, Y., Guo, Z., Fang, J., et al. (2018) The mechanisms of Bushen-Yizhi formula as a therapeutic agent against alzheimer's disease. Sci. Rep., 8, 3104

152. Huang, J., Tang, H., Cao, S., He, Y., Feng, Y., Wang, K. and Zheng, Q. (2017) Molecular targets and associated potential pathways of danlu capsules in hyperplasia of mammary glands based on systems pharmacology. Evid. Based Complement. Alternat. Med., 2017, 1930598

153. Luo, Y., Wang, Q. and Zhang, Y. (2016) A systems pharmacology approach to decipher the mechanism of danggui-shaoyao-san decoction for the treatment of neurodegenerative diseases. J. Ethnopharmacol., 178, 66-81

154. Zheng, C. S., Fu, C. L., Pan, C. B., Bao, H. J., Chen, X. Q., Ye, H. Z., Ye, J. X., Wu, G. W., Li, X. H., Xu, H. F., et al. (2015) Deciphering the underlying mechanisms of Diesun Miaofang in traumatic injury from a systems pharmacology perspective. Mol. Med. Rep., 12, 1769-1776

155. Xu, H., Zhang, Y., Lei, Y., Gao, X., Zhai, H., Lin, N., Tang, S., Liang, R., Ma, Y., Li, D., et al. (2014) A systems biology-based approach to uncovering the molecular mechanisms underlying the effects of dragon's blood tablet in colitis, involving the integration of chemical analysis, ADME prediction, and network pharmacology. PLoS One, 9, e101432

156. Li, H., Zhao, L., Zhang, B., Jiang, Y., Wang, X., Guo, Y., Liu, H., Li, S. and Tong, X. (2014) A network pharmacology approach to determine active compounds and action mechanisms of Ge-GenQin-Lian decoction for treatment of type 2 diabetes. Evid. Based Complement. Alternat. Med., 2014, 495840

157. Liang, X., Li, H. and Li, S. (2014) A novel network pharmacology approach to analyse traditional herbal formulae: the Liu-Wei-Di-Huang pill as a case study. Mol. Biosyst., 10, 1014-1022

158. Yao, Y., Zhang, X., Wang, Z., Zheng, C., Li, P., Huang, C., Tao, W., Xiao, W., Wang, Y., Huang, L., et al. (2013) Deciphering the combination principles of traditional Chinese medicine from a systems pharmacology perspective based on Ma-huang decoction. J. Ethnopharmacol., 150, 619-638

159. Tang, F., Tang, Q., Tian, Y., Fan, Q., Huang, Y. and Tan, X. (2015) Network pharmacology-based prediction of the active ingredients and potential targets of Mahuang Fuzi Xixin decoction for application to allergic rhinitis. J. Ethnopharmacol., $176,402-412$

160. Huang, T., Ning, Z., Hu, D., Zhang, M., Zhao, L., Lin, C., Zhong, L. L. D., Yang, Z., Xu, H. and Bian, Z. (2018) Uncovering the mechanisms of Chinese herbal medicine (MaZiRenWan) for functional constipation by focused network pharmacology approach. Front. Pharmacol., 9, 270

161. Wang, X., Yu, S., Jia, Q., Chen, L., Zhong, J., Pan, Y., Shen, P., Shen, Y., Wang, S., Wei, Z., et al. (2017) NiaoDuQing granules relieve chronic kidney disease symptoms by decreasing renal fibrosis and anemia. Oncotarget, 8, 55920-55937

162. Ke, Z. P., Zhang, X. Z., Ding, Y., Cao, L., Li, N., Ding, G., Wang, Z. Z. and Xiao, W. (2015) Study on effective substance basis and molecular mechanism of Qigui Tongfeng tablet using network pharmacology method. Zhongguo Zhongyao Zazhi, 40, 28372842, in Chinese

163. Tao, W., Xu, X., Wang, X., Li, B., Wang, Y., Li, Y. and Yang, L. (2013) Network pharmacology-based prediction of the active ingredients and potential targets of Chinese herbal Radix Curcumae formula for application to cardiovascular disease. J. Ethnopharmacol., 145, 1-10

164. Yang, H., Zhang, W., Huang, C., Zhou, W., Yao, Y., Wang, Z., Li, Y., Xiao, W. and Wang, Y. (2014) A novel systems pharmacology model for herbal medicine injection: a case using reduning injection. BMC Complement. Altern. Med., 14, 430

165. Luo, F., Gu, J., Zhang, X., Chen, L., Cao, L., Li, N., Wang, Z., Xiao, W. and Xu, X. (2015) Multiscale modeling of drug-induced effects of ReDuNing injection on human disease: from drug molecules to clinical symptoms of disease. Sci. Rep., 5, 10064

166. Liu, J., Sun, K., Zheng, C., Chen, X., Zhang, W., Wang, Z., Shar, P. A., Xiao, W. and Wang, Y. (2015) Pathway as a pharmacological target for herbal medicines: an investigation from reduning injection. PLoS One, 10, e0123109

167. Wu, L., Wang, Y., Nie, J., Fan, X. and Cheng, Y. (2013) A network pharmacology approach to evaluating the efficacy of Chinese medicine using genome-wide transcriptional expression data. Evid. Based Complement. Alternat. Med., 2013, 915343

168. Shen, X., Zhao, Z., Luo, X., Wang, H., Hu, B. and Guo, Z. (2016) Systems pharmacology based study of the molecular mechanism of SiNiSan formula for application in nervous and mental diseases. Evid. Based Complement. Alternat. Med., 2016, 9146378

169. Wang, H. H., Zhang, B. X., Ye, X. T., He, S. B., Zhang, Y. L. and Wang, Y. (2015) Study on mechanism for anti-depression efficacy 
of Sini San through auxiliary mechanism elucidation system for Chinese medicine. Zhongguo Zhongyao Zazhi, 40, 3723-3728, in Chinese

170. Zheng, C. S., Xu, X. J., Ye, H. Z., Wu, G. W., Li, X. H., Xu, H. F. and Liu, X. X. (2013) Network pharmacology-based prediction of the multi-target capabilities of the compounds in Taohong Siwu decoction, and their application in osteoarthritis. Exp. Ther. Med., 6, 125-132

171. Wang, T., Wu, Z., Sun, L., Li, W., Liu, G. and Tang, Y. (2018) A computational systems pharmacology approach to investigate molecular mechanisms of herbal formula Tian-Ma-Gou-Teng-Yin for treatment of alzheimer's disease. Front. Pharmacol., 9, 668

172. Li, Y., Zhang, J., Zhang, L., Chen, X., Pan, Y., Chen, S. S., Zhang, S., Wang, Z., Xiao, W., Yang, L., et al. (2015) Systems pharmacology to decipher the combinational anti-migraine effects of Tianshu formula. J. Ethnopharmacol., 174, 45-56

173. Gao, Y., Gao, L., Gao, X. X., Zhou, Y. Z., Qin, X. M. and Tian, J. S. (2015) An exploration in the action targets for antidepressant bioactive components of Xiaoyaosan based on network pharmacology. Acta Pharma. Sinica (Yao Xue Xue Bao), 50, 1589-1595, in Chinese

174. Liu, J., Pei, T., Mu, J., Zheng, C., Chen, X., Huang, C., Fu, Y., Liang, Z. and Wang, Y. (2016) Systems pharmacology uncovers the multiple mechanisms of Xijiao Dihuang decoction for the treatment of viral hemorrhagic fever. Evid. Based Complement. Alternat. Med., 2016, 9025036

175. Pang, H. Q., Yue, S. J., Tang, Y. P., Chen, Y. Y., Tan, Y. J., Cao, Y. J., Shi, X. Q., Zhou, G. S., Kang, A., Huang, S. L., et al. (2018) Integrated metabolomics and network pharmacology approach to explain possible action mechanisms of Xin-Sheng-Hua granule for treating Anemia. Front. Pharmacol., 9, 165
176. Chen, L., Cao, Y., Zhang, H., Lv, D., Zhao, Y., Liu, Y., Ye, G. and Chai, Y. (2018) Network pharmacology-based strategy for predicting active ingredients and potential targets of Yangxinshi tablet for treating heart failure. J. Ethnopharmacol., 219, 359-368

177. Huang, J., Cheung, F., Tan, H. Y., Hong, M., Wang, N., Yang, J., Feng, Y. and Zheng, Q. (2017) Identification of the active compounds and significant pathways of yinchenhao decoction based on network pharmacology. Mol. Med. Rep., 16, 45834592

178. An, L. and Feng, F. (2015) Network pharmacology-based antioxidant effect study of Zhi-Zi-Da-Huang decoction for alcoholic liver disease. Evid. Based Complement. Alternat. Med., 2015, 492470

179. Li, F., Lv, Y. N., Tan, Y. S., Shen, K., Zhai, K. F., Chen, H. L., Kou, J. P. and Yu, B. Y. (2015) An integrated pathway interaction network for the combination of four effective compounds from ShengMai preparations in the treatment of cardio-cerebral ischemic diseases. Acta Pharmacol. Sin., 36, 1337-1348

180. Li, B., Tao, W., Zheng, C., Shar, P. A., Huang, C., Fu, Y. and Wang, Y. (2014) Systems pharmacology-based approach for dissecting the addition and subtraction theory of traditional Chinese medicine: an example using Xiao-Chaihu-Decoction and Da-Chaihu-Decoction. Comput. Biol. Med., 53, 19-29

181. Zhou, W. and Wang, Y. (2014) A network-based analysis of the types of coronary artery disease from traditional Chinese medicine perspective: potential for therapeutics and drug discovery. J. Ethnopharmacol., 151, 66-77

182. Wang, J., Liu, R., Liu, B., Yang, Y., Xie, J. and Zhu, N. (2017) Systems pharmacology-based strategy to screen new adjuvant for hepatitis B vaccine from traditional Chinese medicine ophiocordyceps sinensis. Sci. Rep., 7, 44788 